\title{
Gestión del riesgo de desastres naturales en la parroquia Pano, Provincia de Napo
}

\author{
Risk Management of natural disasters in Pano parish, Napo province
}

Magali Alexandra Uvidia Vilema. ${ }^{1}$, Angélica Lizeth Chugñay Cargua. ${ }^{2}$, Manfredy Ismael Chugñay Cargua. ${ }^{3}$ \& Luis Eduardo Riofrío López. ${ }^{4}$

\section{Recibido: 14-07-2019 / Revisado: 23-07-2019 /Aceptado: 29-07-2019/ Publicado: 10-09-2019}

\begin{abstract}
DOI: https://doi.org/10.33262/cienciadigital.v3i3.4.874

The Pano parish, of the Napo province due to its location in the east, has a high incidence of floods, especially due to river overflows, in addition to other dangers. To this is added the ignorance of the community in specific procedures to face an emergency and / or disaster of natural and anthropic origin, added to this the probability of occurrence of an adverse event, taking as a main reference floods, as a possible scenario they configure a latent danger in the population that is why a study for Risk Management is proposed for which it is proposed in the investigation chapters that will serve to mitigate the risk of the area.

The purpose of this research is to analyze the vulnerabilities and capacities of the population of the nine communities that make up the Pano parish, Napo province, identifying the factors that have impeded the progress of an immediate response to an adverse event. With the obtaining of these data, strategies can be proposed to strengthen the action capacities linked to the projection of mitigating the risks in the communities that make up the Pano parish.

The methodology that is used is mixed since qualitative and quantitative data is obtained to then perform the respective analysis. Among the results that were found, there is a threat of floods and landslides, the vulnerability of the population can be identified in terms of basic services, and there are different vulnerabilities such as the

\footnotetext{
${ }^{1}$ Escuela Superior Politécnica de Chimborazo, Sede Orellana, Ambiental, Francisco de Orellana, Ecuador, magali.uvidia@espoch.edu.ec

${ }^{2}$ Escuela Superior Politécnica de Chimborazo, IPEC, Riobamba, Ecuador, angelica.chugnay@espoch.edu.ec

${ }^{3}$ Escuela Superior Politécnica de Chimborazo, Sede Orellana, Ambiental, Francisco de Orellana, Ecuador, machugnay@espoch.edu.ec

${ }^{4}$ Consultor Independiente, Iriofrio915@gmail.com
} 
lack of awareness of the risk in which they live; to all this it is necessary to emphasize that both the community leaders and the inhabitants of the communities have the predisposition to receive training and be proactive in the environment that they manage this constituting a strength of the community.

Keywords: Risk management, vulnerability, Parish Pano, Ecuador.

\section{Resumen}

La parroquia Pano, de la provincia Napo por su ubicación en el oriente, tiene una fuerte incidencia de inundaciones en especial por desbordamientos de ríos, además de otros peligros a esto se le suma el desconocimiento de la comunidad en procedimientos específicos para afrontar una emergencia y/o desastre de origen natural y antrópico, sumado a esto la probabilidad de ocurrencia de un evento adverso, tomando como referencia principal inundaciones, como escenario posible configuran un peligro latente en la población es por eso que se plantea un estudio para la Gestión de Riesgo para lo cual se plantea en la investigación capítulos que servirán para mitigar el riesgo de la zona.

El propósito de esta investigación es analizar las vulnerabilidades y las capacidades de la población de las nueve comunidades que conforman la parroquia Pano, provincia de Napo identificando los factores que han obstaculizado el progreso de respuesta inmediata frente a un evento adverso. Con la obtención de estos datos se puede plantear estrategias para fortalecer las capacidades de acción vinculadas con la proyección de mitigar los riesgos en las comunidades que conforman la parroquia Pano.

La metodología que se utiliza es mixta ya que se obtiene datos cualitativos y cuantitativos para luego realizar el respectivo análisis. Dentro de los resultados que se encontraron existe amenaza de inundaciones y deslizamientos, se puede identificar la vulnerabilidad de la población en términos de servicios básicos, además se encuentra diferentes vulnerabilidades como la no concientización del riesgo en el que viven; a todo esto es necesario recalcar que tanto los líderes comunitarios como los habitantes de las comunidades tienen la predisposición de receptar las capacitaciones y ser proactivos en el entorno que se manejan esto constituyéndose en una fortaleza de la comunidad.

Palabras claves: Gestión de riesgos, vulnerabilidad, parroquia Pano, Ecuador.

\section{Introducción}

Las amenazas latentes de deslizamientos e inundaciones en los países del sur del continente han estado presentes durante los últimos años, presentándose desastres que dejan afectaciones tanto económicas como sociales en las personas que habitan en las comunidades que se encuentran en zonas de riesgo.

El riesgo o la probabilidad de daños y pérdidas, es un concepto que supone la existencia de factores tales como amenazas y vulnerabilidades. La idea de amenaza hace referencia a la probabilidad de la ocurrencia de un evento físico dañino para la sociedad; la vulnerabilidad 
se refiere a la propensidad de una sociedad o elemento de la sociedad de sufrir daño (Lavell A., 1999).

La ubicación originaria de un número considerable de los centros urbanos a escala mundial, se explica por su proximidad a diversos recursos naturales como mares, océanos, lagos, ríos o depósitos minerales, valles, faldas volcánicas, entre otras. Sin embargo, por el mismo hecho de ubicación y por el proceso de la naturaleza, los recursos que ofrecen oportunidades para la vida humana, se convierten en distintos momentos en amenazas para ella misma y sus creaciones. Los mares y océanos se convierten en amenazas por la presencia cíclica de huracanes y tormentas tropicales, mareas altas combinadas a veces con descargas fluviales anormales, factores que condicionan la existencia de inundaciones de gran escala, erosión costera y vientos de alta velocidad. Los ríos cíclicamente producen inundaciones que ponen en peligro a la comunidad establecida en sus proximidades, las faldas de los volcanes se transforman en lugares de peligro frente a la posibilidad de la activación volcánica. La tierra es por naturaleza un lugar a veces peligroso, la amenaza natural asociada con los procesos geológicos, geomorfológicos, climáticos y oceanográficos tienden a ser una amenaza constante en términos de un número importante de los centros urbanos grandes, medianos y pequeños del mundo (Lavell A., 1999).

En el Ecuador, la parroquia Pano, de la provincia Napo por su ubicación en el trópico húmedo con una altura que fluctúa entre los 470 y $4920 \mathrm{msnm}$., tiene una fuerte incidencia de inundaciones en especial por desbordamientos de ríos, además de otros peligros tiene una población de 1620 habitantes en 9 comunidades a esto se le suma el desconocimiento de la comunidad en procedimientos específicos para afrontar una emergencia y/o desastre de origen natural y antrópico, sumado a esto la probabilidad de ocurrencia de un evento adverso, tomando como referencia principal inundaciones, como escenario posible configuran un peligro latente en la población es por eso que se plantea un estudio para la Gestión de Riesgo.

\section{Metodología}

\section{Materiales y Métodos}

\section{DISEÑO DE LA INVESTIGACIÓN}

Ya que la Gestión de Riesgos tiene por objeto proporcionar un método para evaluar y mejorar los resultados en la prevención de desastres naturales por medio de la gestión eficaz de los peligros, y debido a que la parroquia Pano, de la provincia de Napo no cuenta con la gestión técnica, para la presente investigación es necesaria la conjunción de métodos de investigación de campo, cualitativos y aplicativos que permitan mediante el uso de diferentes técnicas e instrumentos de recolección de datos la identificación de factores de riesgo a fin de evaluarlos y tomar las acciones enmarcadas a solucionar los problemas detectados.

De esta manera se procederá a la identificación, medición y evaluación de los riesgos presentes en las nueve comunidades de la parroquia Pano además establecer las medidas correctivas y preventivas para la mitigación de los riesgos. La Gestión de riesgo contiene los siguientes elementos: Identificación de riesgos - Evaluación de Riesgos - Acciones Preventivas y Correctivas. 


\section{TIPO DE INVESTIGACIÓN}

La finalidad principal de la presente investigación es disminuir la vulnerabilidad que tienen los habitantes de las comunidades de la parroquia Pano mediante la Gestión de Riesgo que permita proteger y promover la integridad de la población, se utilizó de manera general el Tipo de Investigación aplicada misma que tiene como propósito dar solución a problemas, y; además ya que la información para el desarrollo se la obtendrá directamente de la fuente será también una investigación de campo.

\section{MÉTODOS DE INVESTIGACIÓN}

Descriptiva: El método descriptivo se basa en recoger, organizar, resumir, presentar, analizar, los resultados de las observaciones obtenidos mediante los hechos o fenómenos actuales, identificando así los riesgos a los que están expuesto los habitantes de la parroquia. Este método no se limita a la simple recolección y tabulación de datos, sino que se relaciona con condiciones de vivienda existentes, opiniones de los habitantes, puntos de vista, actitudes que se mantienen para dar una idea clara sobre el problema.

Deductivo: El método Deductivo parte de conocimientos generalizados a fin de ser sintetizados, analizados hacia el logro de un algo con particularidad.

\section{TÉCNICAS E INSTRUMENTOS PARA RECOLECCIÓN DE DATOS}

Se utilizará la técnica de la encuesta y observación directa de procedimientos, conocimiento, capacidades locales e institucionales del territorio aplicando cuestionarios y listas de chequeo (check list).

\section{Observación}

Es una técnica que consiste en observar atentamente el fenómeno, hecho o caso, tomar información y registrarla para su posterior análisis. Es un elemento fundamental de todo proceso investigativo, en ella se apoya el investigador para obtener el mayor número de datos. Gran parte del acervo de conocimientos que constituye la ciencia ha sido lograda mediante la observación.

\section{Encuesta}

Es una técnica de adquisición de información de interés sociológico, mediante un cuestionario previamente elaborado, a través del cual se puede conocer las vulnerabilidades. También será de gran apoyo las siguientes guías metodológicas:

- Análisis de Sistemas de Gestión del Riesgo de Desastres. FAO 2009.

- Manual del Comité de Gestión de Riesgos. Secretaría de Gestión de Riesgos. Ecuador, 2014.

- Metodología para el análisis de vulnerabilidad y riesgo ante inundaciones y sismos, de la edificación de centros urbanos. PREDES, Perú. Lozano O., 2008

\section{Guía de entrevista estructurada}

Se realizó a través de expertos y actores sociales los 9 líderes comunitarios de la parroquia Pano, de la provincia de Napo, autoridades del GAD del Cantón Tena, instituciones que actúan en la zona. Las entrevistas se realizaron con el fin de identificar su conocimiento de las amenazas de deslizamientos e inundaciones, así como las vulnerabilidades sociales 
existentes, su percepción de las capacidades sociales de las familias para enfrentar las amenazas y sobre la creación de estrategias comunitarias para la reducción de riesgos.

Los actores involucrados se han elegido mediante un muestreo por su rol e importancia diagnóstico de vulnerabilidades y capacidades sociales en las comunidades de la parroquia Pano en el área de gestión de riesgos.

\section{POBLACIÓN Y MUESTRA}

De acuerdo con los datos proporcionados por el Censo de Población y Vivienda y proyectados por el Instituto Nacional de Estadísticas y Censos, en el 2015 la parroquia Pano tiene una población aproximada de 1.620 habitantes.

Para el cálculo de la muestra se ha aplicado la fórmula que posteriormente se indica, se ha tomado de la comunidad de Centro Pano por ser el territorio más poblado y cabecera parroquial, concentra la infraestructura y servicios de toda la parroquia. Se asienta sobre un territorio plano y está rodeada del río Pano.

Para la desviación estándar se estima un valor de 0,5 . Se ha considerado el $95 \%$ de nivel de confianza y 5\% de límite aceptable de error por lo que corresponde un valor de $\mathrm{Z}=1,96$ y $\mathrm{e}=0,05$.

El resultado de la aplicación de la fórmula para el cálculo del tamaño de muestra es n=169 personas.

$$
n=\frac{N \sigma^{2} Z^{2}}{(N-1) e^{2}+\sigma^{2} Z^{2}}
$$

Donde:

$\mathrm{n}=$ Número de muestra

N= Tamaño de la población

Z= Nivel de confianza

e= Precisión 5\%

Como la población del Pano es 50\% hombres y 50\% mujeres, los encuestados serán 84 varones y 85 mujeres, escogidos mediante muestreo aleatorio estratificado, repartidos entre las 9 comunidades, 5 instituciones públicas, 3 privadas y 2 Unidades Educativas. Es decir, se encuestarán, 4 varones y 4 mujeres en cada estrato de muestreo, quedando 17 personas por muestrear, las mismas que serán escogidas al azar en el centro poblado de la parroquia.

\section{Resultados}

Con la finalidad de dar a conocer los resultados obtenidos mediante la aplicación de los métodos y técnicas de la presente investigación se ha dividido el análisis e interpretación de resultados en dos etapas; la Inicial que corresponde al análisis enfocado a obtener el diagnóstico situacional de la parroquia Pano con la finalidad de establecer la línea base y la 
etapa Final que corresponde a los resultados posteriores de la ejecución de actividades de mejora y programas correctivos.

\section{Etapa I. Resultados iniciales}

Se parte conociendo que las comunidades de la parroquia Pano, no cuenta con un Plan de Gestión de Riesgo, sin embargo, fue necesario comenzar con una valoración inicial a fin de determinar si existen requerimientos técnicos legales que pueden haberse implementado sin contar con un sistema.

A continuación, se muestra una tabla resumen de los principales resultados obtenidos en la evaluación.

Tabla 1. Resultados evaluación inicial PROCENTAJES DE CUMPLIMIENTO Y NO CUMPLIMIENTO

\begin{tabular}{|c|c|c|c|c|c|c|c|}
\hline ELEMENTOS & 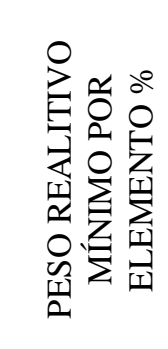 & 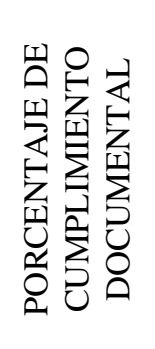 & 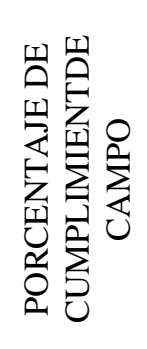 & 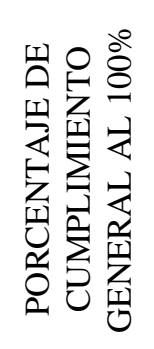 & 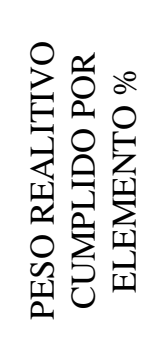 & 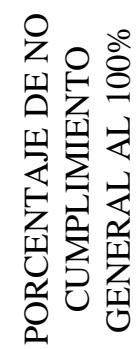 & 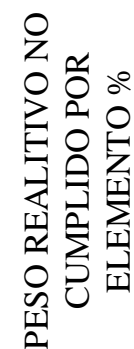 \\
\hline $\begin{array}{l}\text { VALORES } \\
\text { TOTALES }\end{array}$ & $80 \%$ & $0 \%$ & $0 \%$ & $0 \%$ & $13 \%$ & $100 \%$ & $87 \%$ \\
\hline
\end{tabular}

Fuente: Elaboración propia

Los elementos mínimos por cada dimensión de análisis son: una matriz de identificación de riesgos, propuestas de acciones de prevención y mitigación, medidas de autoprotección, plan de contingencias, protocolos de acción y propuestas de presupuestos. Se considera mínimo dos requisitos por cada elemento.

El Porcentaje de Cumplimiento General es del $0 \%$ lo cual quiere decir que las comunidades de la parroquia Pano posee un nivel de cumplimiento ineficiente con una gestión nula en cuanto a seguridad y salud de sus habitantes, para corroborar este dato técnicamente visible se procede a aplicar la fórmula de índice de Eficacia, detallado a continuación:

$$
I E=\frac{N o \text { de requerimientos técnicos legales, integrados/implantados }}{N^{\mathrm{o}} \text { total de requisitos técnicos legales aplicables }} * 100
$$

Donde:

$$
I E=\frac{1}{12} * 100=\mathbf{8} \%
$$

De acuerdo a las variables establecidas y mediante la aplicación de la fórmula correspondiente para conocer el índice de Eficacia se determinó que NO CUMPLE el porcentaje mínimo de gestión $(80 \%)$, datos que son totalmente insatisfactorios. 
Identificación de riesgos

Tabla 2. Resultado de la identificación de riesgos

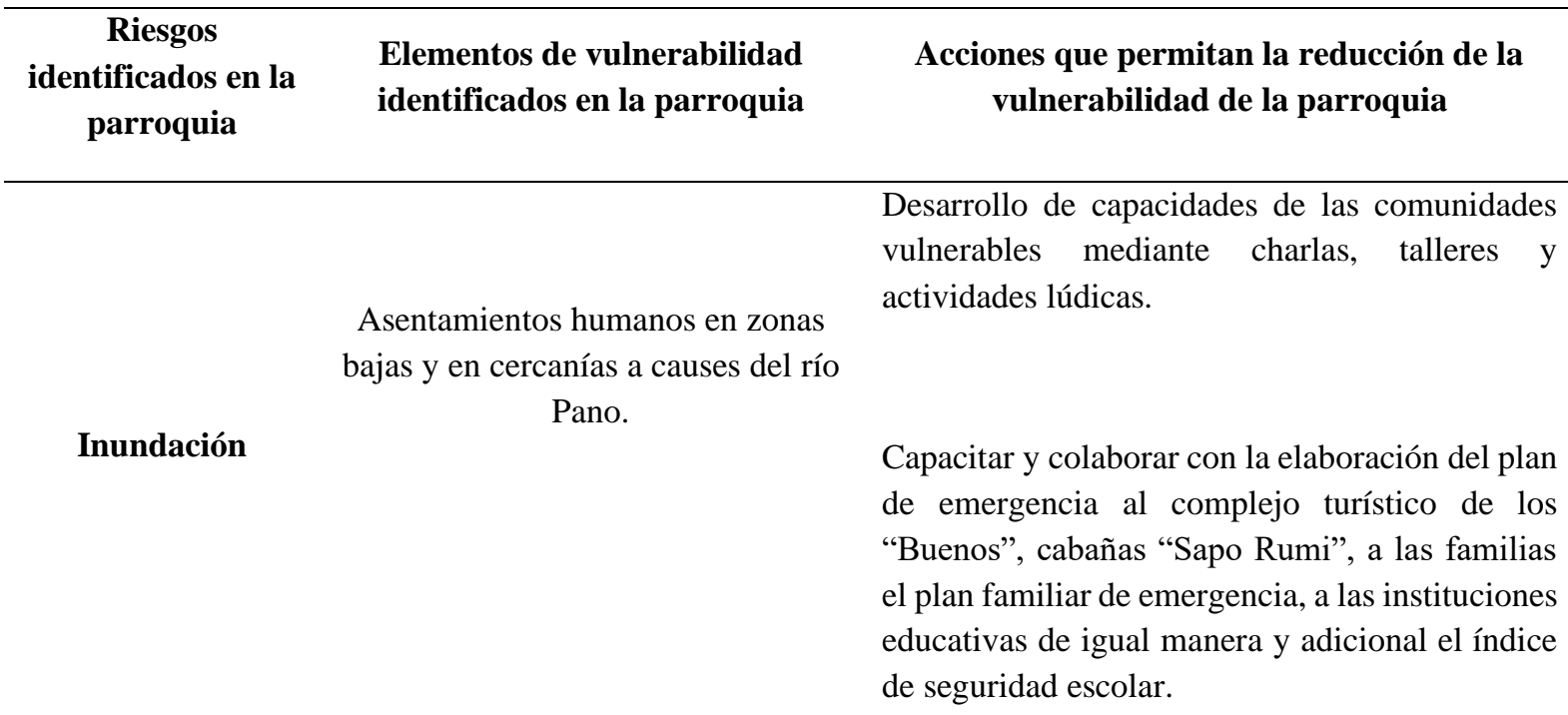

\begin{tabular}{|c|c|c|}
\hline Deslizamiento & Vía Tena-Pano-Talag & Limpieza de vías y cunetas \\
\hline Sismos & 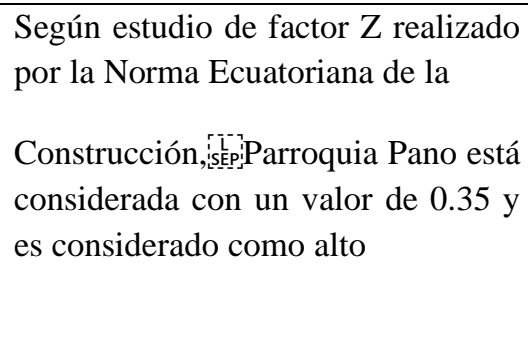 & $\begin{array}{l}\text { Exigencia de normas de sismo-resistencia en la } \\
\text { construcción de nuevas edificaciones en el } \\
\text { territorio. } \\
\text { Preparación de medidas de autoprotección. } \\
\text { Señalética de zonas seguras y áreas de } \\
\text { inundación. }\end{array}$ \\
\hline $\begin{array}{l}\text { Eventos } \\
\text { antropogénicos }\end{array}$ & $\begin{array}{l}\text { Vías de tránsito sin vereda o lugar } \\
\text { seguro del paso del peatón. }\end{array}$ & Mantenimiento y readecuación de puentes \\
\hline
\end{tabular}

Fuente: Elaboración propia

\section{Fortalecimiento de las Capacidades Institucionales en Gestión de Riesgos}

- Apersonamiento, por parte de la presidenta del GAD Parroquial, del compromiso en cumplir y hacer cumplir políticas locales para reducir el riesgo de desastres.

- Acercamiento institucional entre el GAD Parroquial, MIES, tenencia política, IESS y MSP.

- Conformación del Mecanismo de Reducción de Riesgo/Comité de Operaciones de Emergencia. 
- Conformación de brigadas comunitarias.

- Socialización de la Cadena de llamadas para activar el COE.

- Socialización del protocolo de emergencias ante: inundaciones, sismos, incendios y deslizamientos.

- Propuesta de acciones de prevención y mitigación.

- Propuesta de temario para capacitaciones en gestión de riesgos.

- Propuesta de medidas de autoprotección para socializar a la población del territorio vulnerable en especial a las zonas de interés.

- Propuesta de presupuesto básico para dar continuidad a la gestión de riesgos de desastres en el territorio.

- Elaboración de la propuesta de Plan de Contingencias ante Eventos Adversos.

\section{Zonas de Interés Especial}

Tabla 3. Zonas de interés especial.

\begin{tabular}{|c|c|c|}
\hline N.- & SECTOR & RIESGO \\
\hline 1 & Comunidad Guinea Chimbana, sector Kibilina & Inundación \\
\hline 2 & Comunidad Bandio Alonso. Zonas de cultivo & Inundación \\
\hline 3 & Sapo Rumi & Inundación \\
\hline 4 & Lagarto cocha & Inundación \\
\hline 5 & Puka Peña, diagonal al colegio Guillermo Kadle & Deslizamiento \\
\hline 6 & $\begin{array}{l}\text { Puente vehicular-peatonal sobre el río Pano, conecta a } \\
\text { Centro Pano y Taza Urku }\end{array}$ & Atropellamiento \\
\hline 7 & Puente peatonal-vehicular entre Sapo Rumi y Uchuculín & Atropellamiento \\
\hline 8 & $\begin{array}{l}\text { Puente peatonal sobre el río Achiyacu hacia la casa de la } \\
\text { familia Calapucha Alvarado }\end{array}$ & Caída \\
\hline
\end{tabular}

Fuente: Elaboración propia

\section{Análisis de resultados}

Etapa I. Análisis e interpretación de resultados iniciales.

\section{Conciencia del Riesgo}

Referente a conciencia del riesgo, las personas encuestadas en un $46 \%$ dicen que no recuerdan haber presenciado un derrumbe, así mismo $54 \%$ no recuerda haber presenciado una inundación (Anexo 3). Respecto al peligro de derrumbes, el 58\% no tienen conciencia del peligro de derrumbes existente en la zona (Anexo 3) y el $52 \%$ desconoce sobre inundaciones.

\section{Peligro sobre inundaciones}

Esto se puede corroborar con la entrevista realizada al director de riesgos del GAD Tena quien mencionó que efectivamente las personas que habitan en zonas en peligro de derrumbes o inundaciones no son conscientes de ello, ni tampoco de las consecuencias que traería una inundación como son las enfermedades. 
“...el desconocimiento tiene cierto grado de vulnerabilidad, además el desconocer el peligro al que están expuestos, ya que una inundación trae problemas colaterales, como pueden ser las infecciones, enfermedades del estómago y más patologías que conlleva las inundaciones"

\section{Capacitaciones recibidas}

En lo que concierne a las capacitaciones recibidas en la zona, de las encuestas válidas, el $88 \%$ de los encuestados afirma no haber recibido capacitación sobre derrumbes (Anexo 2). Consideran también en un $92 \%$ que no se consideran capacitados sobre qué hacer si hay un derrumbe (Anexo 2). Esto lo podemos complementar con el resultado obtenido en el grupo focal donde confirmaban no haber tenido capacitaciones amplias sobre estos temas.

\section{Caracterización de Capacidades}

Respecto al tema de caracterización de capacidades sociales a pesar que las familias no han recibido capacitaciones sobre deslizamientos e inundaciones, tienen la predisposición de capacitarse para responder ante alguna emergencia, cuentan también con instituciones comprometidas que están emprendiendo proyectos de capacitación en gestión de riesgo para la comunidad con el fin de contribuir a la vulnerabilidad social.

Se puede comparar con la teoría de Lavell sobre el riesgo que explica que el riesgo puede ser anticipado, pero asimismo permite que la sociedad intervenga en la prevención y mitigación del mismo. (Alan Lavell, 2004). Entonces si como antecedente se tiene el reconocimiento por parte de las autoridades de la grave amenaza en el sector, es necesario fortalecer desde las capacitaciones y organización comunitaria.

\section{Discusión}

Cardona O. (2008) ha agrupado el conjunto de elementos, medidas y herramientas en 4 dimensiones a) identificación del riesgo b) reducción del riesgo c) manejo de desastres y d) gobernabilidad y protección financiera, para intervenir ya sea en la amenaza o en la vulnerabilidad. Los resultados de esta investigación recaen dentro de cada una de las dimensiones antes mencionadas, cuyo fin es la de mitigar los riesgos y dar una mejora continua a la gestión del riesgo de desastres que se está efectuando en el territorio parroquial Pano.

Tabla 4. Resultados de dimensiones

\begin{tabular}{ccl}
\hline N.- & Dimensión & \multicolumn{1}{c}{ Resultados } \\
\hline 1 & Identificación de riesgos & $\begin{array}{l}\text { Matriz de identificación de riesgos } \\
\text { Propuesta de acciones de prevención y mitigación. } \\
2\end{array}$ \\
Reducción de riesgos & $\begin{array}{l}\text { Propuesta de medidas de autoprotección } \\
\text { Plan de contingencias }\end{array}$ \\
3 & Manejo de desastres & Protocolo de inundación \\
4 & Gobernabilidad & $\begin{array}{l}\text { Propuesta de presupuesto básico para dar continuidad a la gestión de } \\
\text { riesgos de desastres en el territorio }\end{array}$ \\
\hline
\end{tabular}

Fuente: Elaboración propia 
Todos estos elementos en conjunto tributan de manera positiva en la reducción de la vulnerabilidad institucional del Gobierno Autónomo Descentralizado Parroquial. La propuesta de un plan de contingencia da seguimiento a la dura tarea de reducir el riesgo de desastres en el territorio Pano e inculca en la población y en la institución la gestión proactiva del riesgo (Cardona O., 2008).

Se observa la misma tendencia de reducción de vulnerabilidad en el trabajo de investigación propuesto por Correa Mayra y Granda José (2013) en el cual concluyen que los actores clave en la gestión del riesgo de desastre es el GAD Parroquial y el Cuerpo de Bomberos. En la parroquia Pano no existe un departamento de bomberos, el más cercano se encuentra ubicado en el cantón Tena a 10 minutos de distancia, por lo que el GAD gestionará con las instituciones públicas y privadas la capacitación continua del COE y habitantes del territorio.

\section{Etapa II. Cumplimiento de los requisitos técnicos legales de la Gestión de Riesgo}

En manifiesto del panorama inicial del presente proyecto en el cual se identificó que las comunidades de la parroquia Pano no contaba con Gestión de Riesgo y su índice de eficacia posterior del análisis del mismo fue del $8 \%$, con la finalidad de conocer el avance en materia de cumplimiento de los requisitos técnico legales que es el ser del presente proyecto, se procede a realizar la autoevaluación de cierre.

A continuación, se muestra una tabla resumen de los principales resultados obtenidos en la evaluación. El Informe final en cual recaba todos los elementos involucrados se lo detalla en el instrumento

Tabla 5. Resultados de la evaluación final de cumplimiento

\begin{tabular}{|c|c|c|c|c|c|c|c|c|}
\hline \multirow[b]{2}{*}{ ELEMENTOS } & \multicolumn{8}{|c|}{ PROCENTAJES DE CUMPLIMIENTO Y NO CUMPLIMIENTO } \\
\hline & 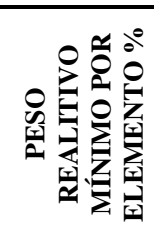 & 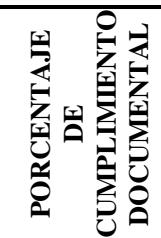 & 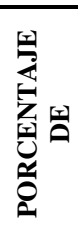 & 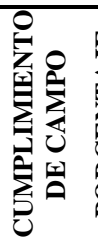 & 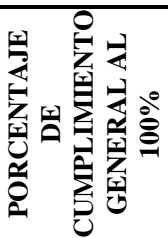 & 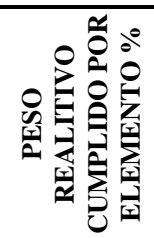 & 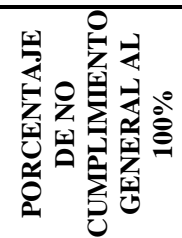 & 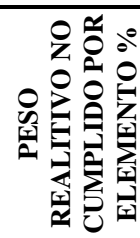 \\
\hline $\begin{array}{l}\text { VALORES } \\
\text { TOTALES }\end{array}$ & $80 \%$ & $95 \%$ & $87 \%$ & & $91 \%$ & $89 \%$ & $9 \%$ & $11 \%$ \\
\hline
\end{tabular}

Fuente: Elaboración propia

Al observar los resultados se puede decir que el porcentaje de cumplimiento general es del $91 \%$ lo cual quiere decir que la población de las comunidades de la parroquia Pano posee un nivel de cumplimiento eficiente con una gestión enmarcada dentro de los parámetros seguridad y salud ocupacional, para corroborar este dato técnicamente visible se procede a aplicar la fórmula de índice de Eficacia del Sistema de Seguridad y Salud Ocupacional, detallado a continuación:

$$
I E=\frac{N \text { o de requerimientos técnicos legales, integrados }- \text { implantados }}{N \text { o total de requisitos técnicos legales aplicables }} * 100
$$

Donde: 


$$
I E=\frac{10}{12} * 100=\mathbf{8 3} \%
$$

De acuerdo a las variables establecidas y mediante la aplicación de la fórmula correspondiente para conocer el índice de Eficacia se determinó que la parroquia Pano SI CUMPLE con los requisitos técnicos en un $83 \%$.

\section{Conclusiones.}

- Los habitantes de la parroquia Pano, de la provincia de Napo es una población vulnerable socialmente, existe un nivel básico de educación, hay desempleo, desconocimiento del nivel de riesgos a deslizamientos al que están expuestos, a esto se suma la falta de alcantarillado, agua potable y demás servicios básicos para esta comunidad.

- En cuanto a las capacidades, existe un bajo nivel de conciencia sobre el peligro, el riesgo y las medidas para prevenirlo y mitigarlo, desde este punto la comunidad se sitúa en un contexto débil de participación comunitaria por su escaso sentido de pertenencia, participación y liderazgo.

- Se destaca que la población como método para superar estas vulnerabilidades tiene la disposición de capacitarse respecto a temas de: deslizamientos, inundaciones, gestión de riesgo y emprendimientos sociales comunitarios.

\section{Referencias bibliográficas.}

Cardona, O. (2008). Medición de la gestión del riesgo en América Latina. Revista Internacional de Sostenibilidad, Tecnología y Humanismo (3).

FAO. (octubre de 2009). Organización de las Naciones Unidas para la Agricultura y la Alimentación. Recuperado el 2017, de http://www.fao.org/3/a-i0304s.pdf

FAO. (s/f). El Ecuador: Un país con elevada vulnerabilidad. Desastres naturales y tenencia de la tierra.

Gobierno Autónomo Descentralizado Parroquial Rural de Pano. (2015). Plan de Desarrollo y Ordenamiento Territorial. Pano.

Lavell, A. (2004). The Lower Lempa River Valley. El Salvador: Risk Reduction and.

Lavell, A. (1999). Gestión de riesgos ambientales urbanos. Red de Estudios Sociales en Prevención de Desastres em América Latina, Facultad Latinoamericana de Ciencias Sociales.

Secretaría de Gestión de Riesgos. (2015). Manual de Evaluación Inicial de Necesidades par Eventos Adversos. 
PARA CITAR EL ARTÍCULO INDEXADO.

Uvidia Vilema, M., Chugñay Cargua, A., Chugñay Cargua, M., \& Riofrío López, L. (2019). Gestión del riesgo de desastres naturales en la parroquia Pano, Provincia de Napo. Ciencia Digital, 3(3.4.), 289-300. https://doi.org/10.33262/cienciadigital.v3i3.4.874

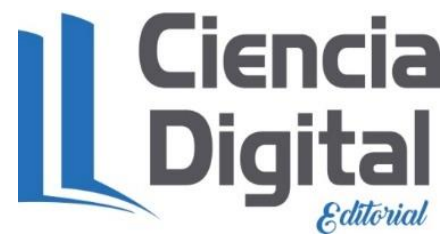

El artículo que se publica es de exclusiva responsabilidad de los autores y no necesariamente reflejan el pensamiento de la Revista Ciencia Digital.

El artículo queda en propiedad de la revista y, por tanto, su publicación parcial y/o total en otro medio tiene que ser autorizado por el director de la Revista Ciencia Digital.
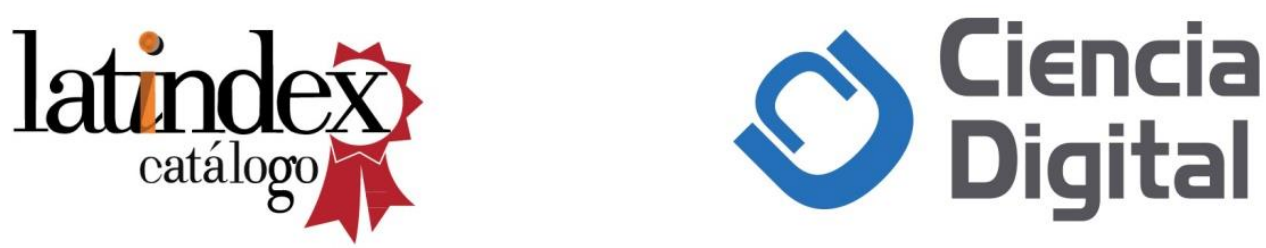